\title{
Characteristic properties of peaks in GRBs
}

\author{
F. Quilligan, K.J. Hurley, B. McBreen, L. Hanlon, and P. Duggan \\ Department of Experimental Physics, University College Dublin, Belfield, Dublin 4, Ireland
}

Received December 29, 1998, accepted March 10, 1999

\begin{abstract}
The brightest 200 GRBs with duration $T_{90}>2 \mathrm{~s}$, selected from the BATSE $4 \mathrm{~B}$ catalog, have been analysed, using a wavelet technique. The characteristics of statistically significant peaks including the rise time, fall time, FWHM, peak height, peak area, time intervals between peaks and the number of peaks per burst have been analysed. The results presented show that the distribution of the areas of the peaks is compatible with a lognormal distribution.

The properties of the peaks before and after the strongest peak have also been compared, and we have analysed the similarities and differences between the pre-peak and post-peak sections of the burst.

The overall properties of peaks in GRBs provide considerable constraints on models that attribute the variability to interactions with an external medium or to the activity of a central engine.
\end{abstract}

Key words: gamma — rays: bursts — methods: data analysis — cosmology: observations

\section{Introduction}

GRBs produce an immense amount of energy $\left(\approx 10^{51} \mathrm{ergs}\right)$ which must be emitted by a medium with highly relativistic velocities $(\gamma>100)$. Most GRBs are highly variable with a variability scale much smaller than their overall durations and there is considerable debate as to the cause of the variability, which may be due to internal or external shocks (e.g. Sari \& Piran 1997; Chiang \& Dermer 1997).

GRBs generally have complex time profiles and previous work (McBreen et al. 1994; Hurley et al. 1997) has indicated that some parameters are consistent with lognormal distributions e.g. durations of GRBs. Li \& Fenimore (1996) extended the analysis to include BATSE GRBs

Send offprint requests to: fquillig@bermuda.ucd.ie

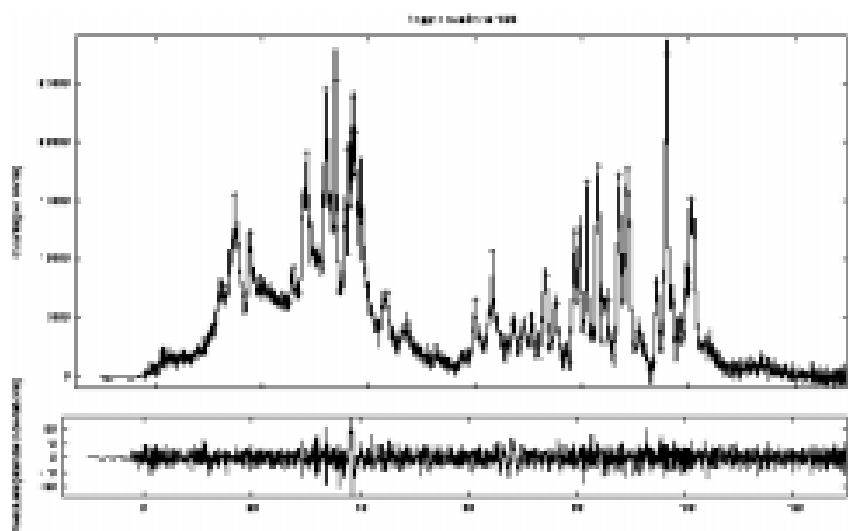

Fig. 1. Trigger No. 1606: Original (solid line) and reconstructed (dashed line) time history are almost indistinguishable. The peaks identified by the algorithm are marked

with multiple peaks and confirmed the applicability of lognormal distributions in GRBs. The results presented here on a large sample of BATSE GRBs were obtained using wavelets. Other techniques have also been used to profile the time structure of GRBs (e.g. Norris et al. 1996; Stern et al. 1996).

\section{Basics of wavelets}

Wavelets facilitate the combination of frequency domain and spatial domain representations of a signal. The technique allows the treatment of signals which are unsuitable for traditional fourier signal analysis - generally because such assumptions as continuity and stationarity are not required. The name wavelet is meant to suggest the wave packet, which is well localised in both spatial and frequency domains simultaneously. Wavelet analysis was pioneered by Daubechies (1992) (see mathematical text by e.g. Meyer 1993). Further information on the our wavelet analysis is presented elsewhere (Hurley et al. 1997). 


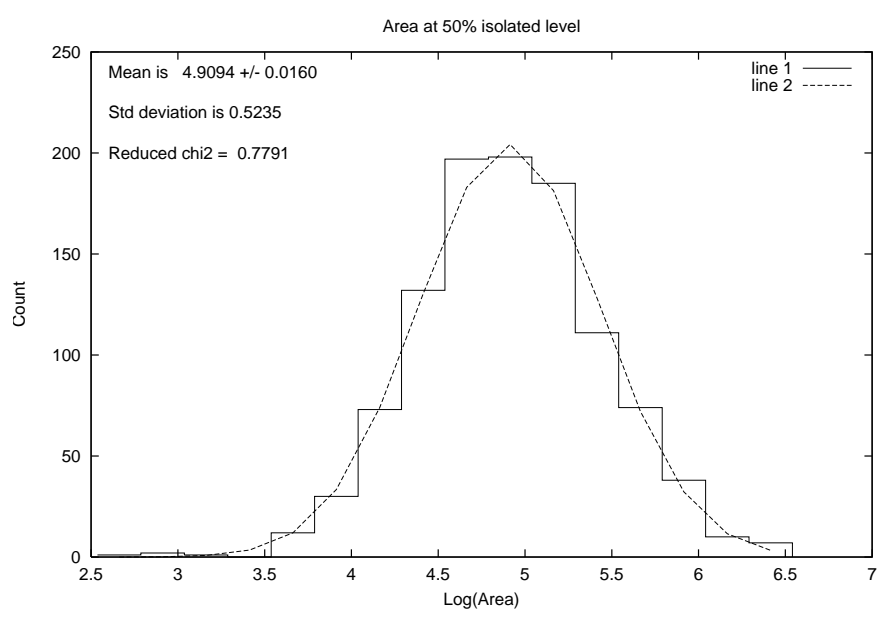

Fig. 2. Distribution of areas under the peaks in brightest 200 GRBs from the BATSE 4th catalog. The dashed line is a good lognormal fit to the distribution

\section{Method}

Following on the wavelet denoising of the original signal the peak structures within the burst were identified. This method is similar to the technique of Li \& Fenimore (1996). Starting at the beginning of the burst we examine each peak and attempt to find minima on each side which are separated from the maximum by more than a chosen significance level. If the search for minima fails on a peak it is rejected and the search for peaks continues. The algorithm is designed such that the minima finally selected for a given peak are the lowest possible choices.

We further extend the analysis to allow the identification of peaks which are well separated or isolated. The technique is one of simply determining the fraction of the total height which is above the higher minima and then applying a threshold on this fraction, above which peaks are considered isolated.

\section{Results}

The distribitions of the peak areas, peak amplitudes and the rise times and fall times are presented in Figs. 2-4. The peak area is quite compatible with a lognormal distribution as shown in Fig. 2.

\section{Conclusions}

A comprehensive profile of the temporal properties of intense GRBs has now been provided by the wavelet method. In particular distributions have been presented for some characteristics of GRBs. The area under the peak is consistent with a lognormal distribution.

A comparison of the pre-peak and post-peak sections of the burst has been completed. A preliminary comparison of the two distributions reveal some significant differences between the pre-peak and post-peak sections of the burst (Quilligan et al. 1999).

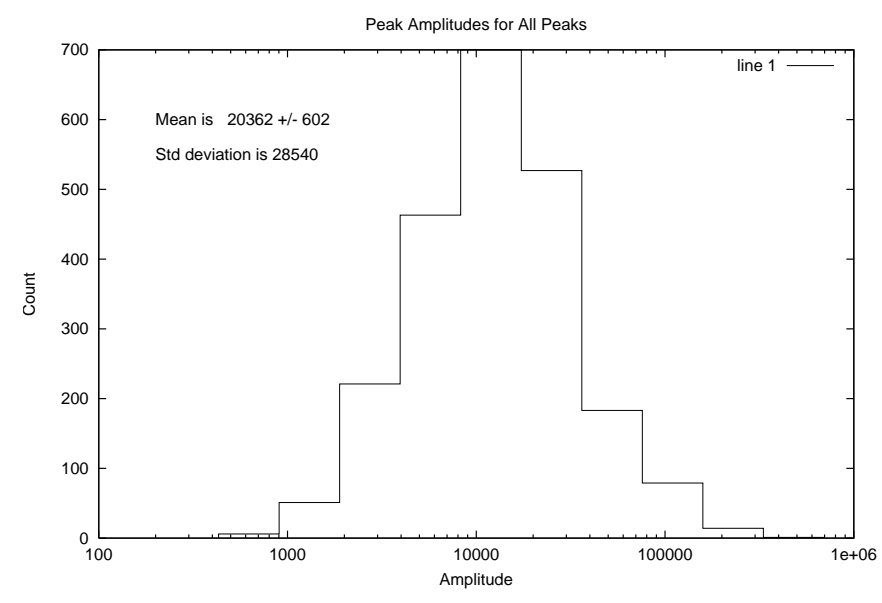

Fig. 3. Distribution of peak amplitudes in brightest 200 GRBs

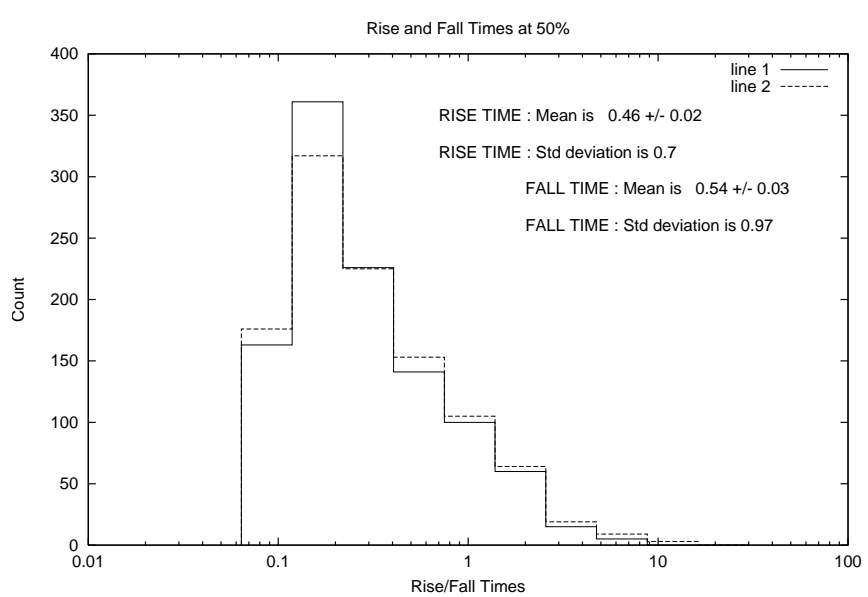

Fig. 4. Distribution of rise (solid) and fall (dotted) times for the peaks in brightest 200 GRBs. The cutoff at short timescales is due to the 64 millisecond time resolution of BATSE

These results provide an alternative description of GRB profiles to that of the peak-aligned stretched exponentials (Stern et al. 1996).

\section{References}

Chiang J., Dermer C.D., 1997, astro-ph/9708035

Daubechies I., 1992, Ten Lectures on Wavelets, SIAM, Philadelphia

Fishman G.J., Meegan C.A., 1995, ARA\&A 33, 415

Hurley K.J., McBreen B., Quilligan F., Delaney M., Hanlon L., 1997, Gamma Ray Bursts, 4th Huntsville Symp, AIP Conf. Proc. 428, 191

Li H., Fenimore E.E., 1996, ApJ 469, L115

McBreen B., Hurley K.J., Long R., Metcalfe L., 1994, MNRAS 271,662

Meyer Y., 1993, Wavelets: Algorithms and Applications, SIAM, Philadelphia, p. 101

Norris J.P., et al., 1996, ApJ 459, 393

Quilligan, et al., 1999 (in preparation)

Sari R., Piran T., 1997, astro-ph/9701002

Stern B.E., Svensson R., 1996, ApJ 469, L109 